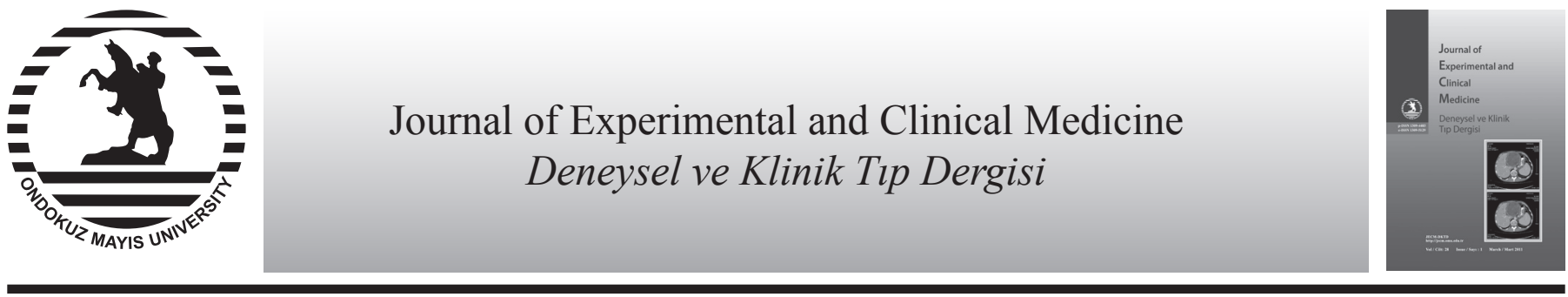

Case Report

\title{
Gastric cancer with a breast metastasis
}

\author{
Güzin Gönüllü*a, Bilge Can ${ }^{b}$, Müge Karaoğlanoğlu ${ }^{a}$, Gamze Dönmez ${ }^{\text {, İdris Yücel }}{ }^{b}$ \\ ${ }^{a}$ Department of Medical Oncology, Medical Faculty, Ondokuz Mayis University Samsun, Turkey \\ ${ }^{b}$ Department of Pathology, Medical Faculty, Ondokuz Mayis University, Samsun, Turkey
}

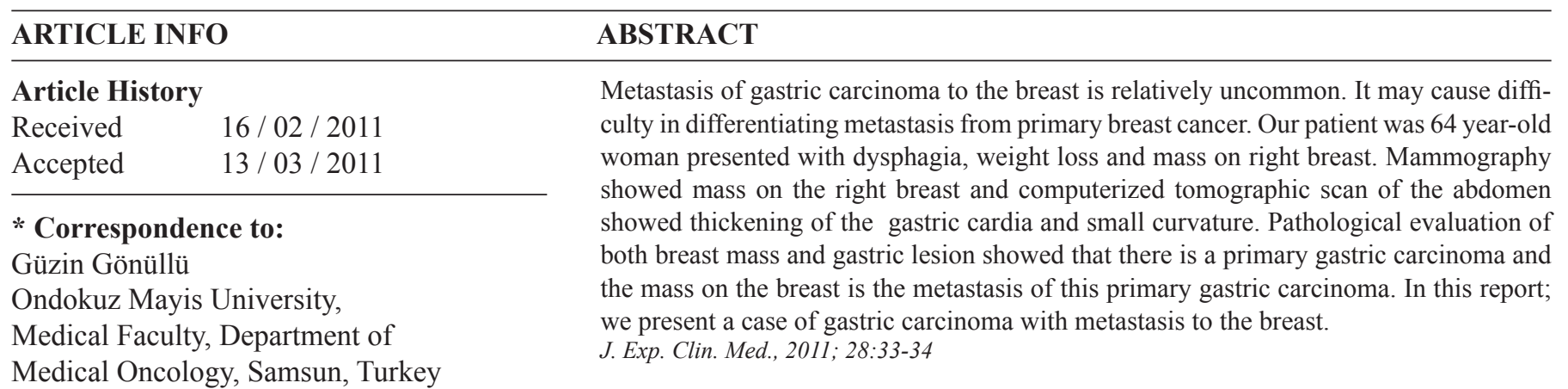

e-mail:ggonullu@omu.edu.tr

\section{Keywords:}

Breast

Cancer

Gastric

Metastasis

(C) 2011 OMU All rights reserved

\section{Introduction}

Breast metastases from other tumors are extremely rare and constituting $2 \%$ of all breast cancers (Yeh et al., 2004). The most primary malignancies metastasizing to the breast are as follows; malign melanom, lung tumors, carcinoid tumors, ovarian tumors, renal cell tumors and gastrointestinal tumors (Boutis et al., 2006). We report a rare case of gastric adenocarcinoma metastasizing to the breast.

\section{Case Report}

In June 2006, a 64-year-old female presented with weight loss and mass on the right breast. Physical examination revealed a mass on the right breast. Mammography showed well-circumscribed, 9 and $8 \mathrm{~mm}$ diamaters increased density in the lower quadrant of the right breast. Microscopic examination of the excisional biopsy of the breast revealed welldifferentiated adenocarcinoma with surrounding hyaline-fibrous stroma. The neoplastic glands of adenocarcinoma were variably sized and had crowded nuclei with hyperchromatism and pleomorphism (Fig. 1a,b). On immunohistochemistry, the neoplastic cells were strongly positive for pan-CK (antihuman cytokeratin, reacting to a wide range of cytokeratin), epithelial membrane antigen and CEA. Estrogen receptors (ER, Clone SP1, Neomarkers) and progesterone receptors (PR, Clone 1A6, Neomarkers) were negative. Mucicarmine revealed the intracytoplasmic mucin in tumor cells. Computerized tomographic scan of abdomen showed thickening of the gastric cardia and small curvature, around superior mesenteric arter pathologic lymph nodes. Upper gastrointestinal endoscopy demonstrated ulserovegetan mass of which obstructed lumen in cardia.

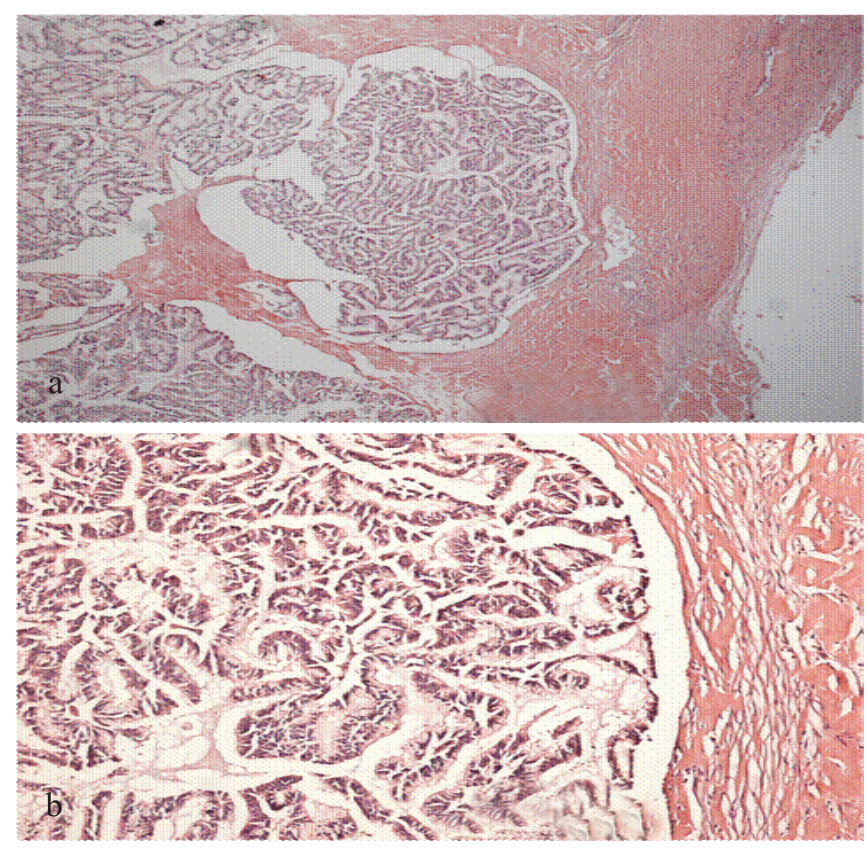

Fig. 1a,b Breast biopsy showing adenocarcinoma with prominent glandular structures (H\&E, x25 and x100). 
Histopathology of gastric biopsy specimens showed a well-differentiated intestinal-type gastric adenocarcinoma with papillary features (Fig. 2).

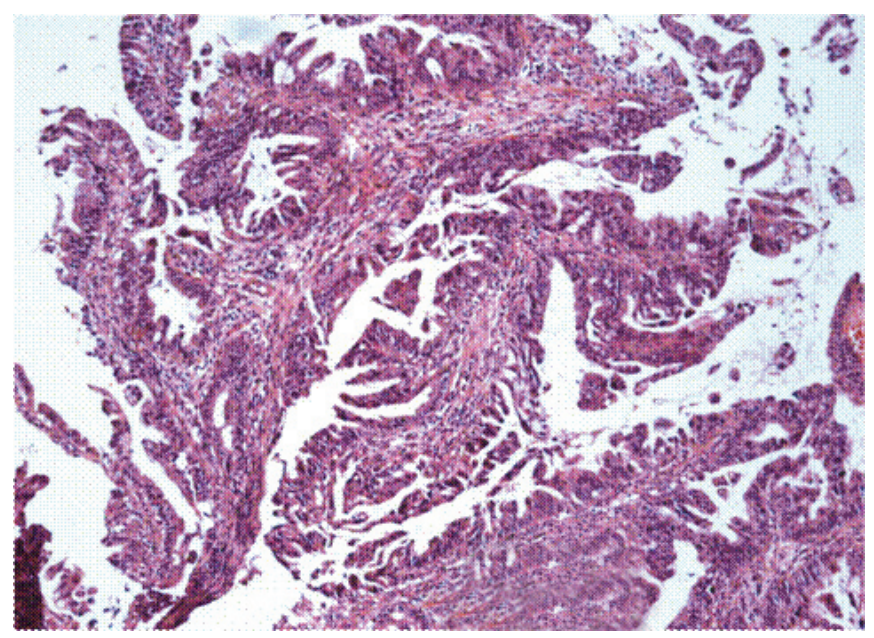

Fig. 2. Gastric biopsy showing intestinal-type gastric adenocarcinoma (H\&E x100).

The mucin and the immunohistochemical staining profile of the stomach carcinoma were identical of the breast tumor. The case was diagnosed as gastric adenocarcinoma metastasis to breast by the help of clinical, radiological and pathological evaluations. After systemic treatment, the patient is still living for 13 months.

\section{Discussion}

Breast metastases from extra-mammary malignancies are in- frequent. The incidence is 1.7- 6.6 \% in autopsy series (Boutis et al., 2006). An accurate diagnosis of breast metastasis is important because the treatment and outcome of primary and secondary malignancies of the breast are completely different (Yeh et al., 2004). Metastatic lesions are generally palpable. Multiple, diffuse, bilateral axillary lymph node involvement are quite rare (Alexander et al., 1989; Hamby et al., 1991; Cavazzini et al., 1993). The clinical presentation is the same for both types of tumors, it is not possible to clinically differentiate a primary breast tumor from a breast metastasis . Our patient presented with only breast mass without gastric complaints. On mammography, the metastatic lesions may appear as well circumscribed round, therefore difficult to distinguish from fibroadenoma and other benign solid lesions. Spicules and microcalcifications are absent in metastatic lesion except metastatic ovarian cancer (psammoma bodies). On mammography; the presence of spiculer lesions and microcalcifications is consistent with primary breast carcinoma (Kwak et al., 2000; Qureshi et a., 2005). The mammograpic findings of our patient is also similar to these findings. The treatment recommendation for gastrointestinal metastasis to breast cancer is systemic treatment of the metastatic disease. The response rate in one series was approximately 30\% and survival exceeding 1 year has been reported (Tremblay et al., 2002). Surgical treatment must be reserved for patients who develop a complication such as obstruction or hemorrhage from the tumor.

Consequently; the onset of gastrointestinal symptoms in a patient with a history of breast mass should prompt the physician to rule out the possibility of breast metastases from primary gastric carcinoma.

\section{REFERENCES}

Alexander, H.R., Turnbull, A.D., Rosen, P.P. 1989. Isolated breast metastases from gastrointestinal carcinomas: report of two cases. J. Surg. Oncol. 42, 264-266.

Boutis, A.L., Andreadis, C., Patakiouta, F., Mouratidou, D. 2006. Gastric signet-ring adenocarcinoma presenting with breast metastasis. World J. Gastroenterol. 12, 2958-2961.

Cavazzini, G., Colpani, F., Cantore, M., Aitini, E., Rabbi, C., Taffurelli, M., 1993. Breast metastasis from gastric signet ring cell carcinoma, mimicking inflammatory carcinoma. A case report. Tumori. 79, 450-453.

Hamby, L.S., McGrath, P.C., Cibull, M.L., Schwartz, R.W. 1991. Gastric carcinoma metastatic to the breast. J. Surg. Oncol. 48, 117-121.

Kwak, J.Y., Kim, E.K., Oh, K.K. 2000. Radiologic findings of metastatic signet ring cell carcinoma to the breast from stomach. Yonsei Med. J. 41, 669-672.

Qureshi, S.S., Shrikhande, S.V., Tanuja, S., Shukla, P.J. 2005. Breast metastases of gastric signet ring cell carcinoma: a differential diagnosis with primary breast signet ring cell carcinoma. J. Postgrad. Med. 51, 125-127.

Tremblay, F., Famison, B., Meterissian, S. 2002. Breast Cancer Masquerading as a Primary Gastric Carcinoma J. Gastrointest. Surg. 6, 614616.

Yeh, C.N., Lin, C.H., Chen, M.F. 2004. Clinical and ultrasonographic characteristics of breast metastases from extramammary malignancies. Am. Surg. 70, 287-290. 